\title{
EVALUACION DEL FACTOR CENTRAL Y PERIFERICO EN FATIGA MUSCULAR EN PACIENTES COM DANNO PIRAMIDAL
}

\author{
CARLOS G. SCHUTZ \\ MARCELA E. PANIZZA \\ L. G. COHEN \\ R. $R E Y$ \\ ULGA P. SANZ
}

\begin{abstract}
Con el objeto de estudiar algunos de los mecanismos de fatiga muscular que se producen en sujetos con daño de la vía piramidal, se dispuso su investigación en un grupo de pacientes hemiparéticos, utilizando la técnica descripta en un trabajo previo ${ }^{4}$.
\end{abstract}

\section{MATERIAL Y METODOS}

Se estudiaron 15 pacientes, 4 mujeres y 11 hombres, cuyas edades oscilaron entre 14 y 70 años, y que presentaban hemiparesia faciobraquiocrural derecha o izquierda, de grado leve a moderado, cuya evolución osciló entre 1 mes a 1 año después de la instalación del cuadro en el momento de la exploración, y cuyas etiologías fueron isquémica o hemorrágica. Los estudios de fatiga se realizaron, tanto en el pie parético como en el sano. Controles: Se utilizó como control, una población de 13 sujetos sanos, 10 hombres y 3 mujeres, voluntarios de este Servicio, y cuyas edades oscilaron entre 25 a 42 años. Métodos: Los detalles de la técnica empleada, fueron dados en un trabajo previo 4.

\section{RESULTADOS}

1. Onda $M$ máxima - En el pie parético, la onđa $M$ máxima final disminuyó al 94.76 $\pm 11.25 \%$ de su valor inicial. En el pie normal, la onda M máxima final disminuyó al $97.35 \pm 7.51 \%$ de su valor inicial. No habiendo entre estas disminuciones diferenciais significativas con lo sufrido por la onda M máxima del grupo testigo que disminuyó al $92.59 \pm 11.37 \%$ de su valor inicial.

2. Frecuencia de los potenciales positivos y negativos que integran el EMG - La frecuencia de los potenciales positivos y negativos que integran el EMG disminvyó al $59.27 \pm 27.76 \%$ y $50.98 \pm 19.49 \%$ respectivamente en relación a los valores iniciales para el pie parético y $62.27 \pm 23.39 \%$ y $63.00 \pm 23.96 \%$ para el pie normal. En tanto que en el grupo control, la disminución fue de $85.05 \pm 45.25 \%$ y $96.14 \pm 58.16 \%$. Lo cual resulta estadisticamente significativo, solamente en el caso de la frecuencia negativa del pie parético $(p<0.005)$, y en ambas frecuencias del normal $(p<0.025$ y $p<0.01$.

Sección de Electroneurofisiología Clínica, Hospital Ramos Mejía. Buenos Aires, Argentina. 
3. Duración de los potenciales positivos y negativos que integran el EMG - La duración de los potenciales positivos y negativos que integran el EMG, aumento al $155.13 \pm 37.63 \%$ y $102.97 \pm 43.75 \%$ respectivamente, con respecto a los valores iniciales para el pie parético, y a $106.70 \pm 24.53 \%$ y $119.03 \pm 6.92 \%$ para el pie normal, con respecto a los valores finales del grupo control: $108.35 \pm 49.29 \%$ y $117.13 \pm 39.82 \%$, no habiendo significación estadística em ningún caso.

\section{COMENTARIOS}

La diferencia entre a y b en el cuadro de las frecuencias (fig. 1) puede ser explicada por una disminución en la frecuencia de batido de las unidades motoras, lo cual a su vez, podría deberse a una disminución de la cantidad de unidades motoras reclutadas en el esfuerzo, ya que es un hecho conocido el que se reduzca el número de unidades motoras funcionantes en la espasticidad ${ }^{6}$ así como en la vejez ${ }^{1}$ o a que las unidades motoras aún en número normal, baten con menor frecuencia, fenómeno también descripto, tảnto en espásticos 5 como en ancianos 7 o a la combinación de ambos.

Es de destacar el hecho de que si bien nuestra población probante, no tiene un promedio de edad mayor de 60 años (grupo etario en el que demos-
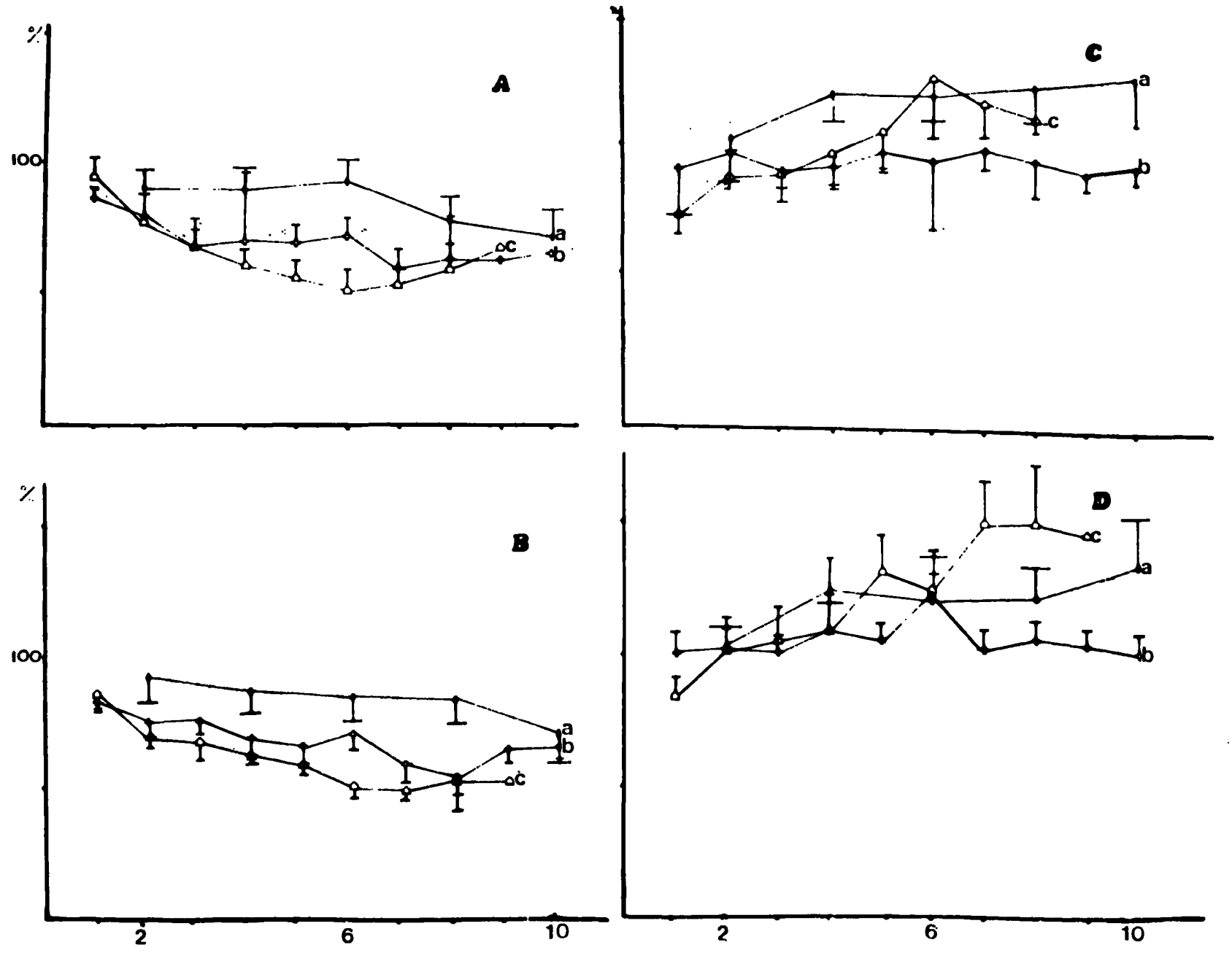

Fig. 1 - A. Frecuencia de potenciales; a: controles, b: lado normal, c: lado parético. $B$. Frecuencia de potenciales; $a$ : controles, b: lado normal, c: lado parético. C. Duración de potenciales; a: controles, b: lado normal, c: lado parético. D. Duración de potenciales; a: controles, b: lado normal, c: lado parético. 
traron claramente los fenómenos antedichos) tiene si, una media considerablemente mayor que la población testigo, lo cual podría contribuir en la presentación de los fenómenos mencionados. Las diferencias entre b y c de la misma figura, serian entonces atribuibles a una mayor pérdida de unidades motoras y/o a un mayor enlentecimiento en la frecuencia de batido de las mismas, en el lado parético en relación al contralateral. Sin embargo, las 3 curvas $(a, b$, c) siguen el mismo patrón de fatiga, es decir, disminución de la frecuencia en función del tiempo de esfuerzo realizado.

El comportamiento de las duraciones (fig. 1), similar en el grupo parético y en el control, podría tener dos explicaciones: 1) que la cantidad de unidades motoras que se suman temporalmente, sea la misma que en sujeitos normales, o 2) que la cantidad de unidades motoras que se suman temporalmente, sea menor; pero compensado por un mayor tamaño de las unidades motoras remanentes, debido a la colateralización axonal y reinervación de fibras musculares previamente denervadas, hecho que sucede tanto en lesiones piramidales ${ }^{2}$, como en el envejecimiento ${ }^{2}$ y que podría compensar la disminución de las unidades funcionantes. La permanencia de la onda $M$ máxima dentro de valores estables al inicio y final del experimento, muestra que a lo largo de él, no ha habido pérdida de unidades motoras ni de fibras musculares individuales dentro de las mismas.

En definitiva, los hallazgos hechos, sugieren que fuera de las diferencias notadas y que pueden ser explicadas por factores ya conocidos, no existen mayores variaciones en relación a los sujetos en cuanto a los mecanismos de fatiga analizados por este método en pacientes portadores de una lesión piramidal.

\section{RESUMEN}

Para evaluar algunos de los componentes de la fatiga muscular en el daño piramidal, se seleccionó un grupo de 15 pacientes con hemiparesia faciobraquiocrural de severidad variable, los cuales fueron sometidos a un esfuerzo muscular, durante el cual se evaluaron los cambios de la frecuencia y duración de los potenciales positivos y negativos del EMG, así como los sufridos por la onda $M$ máxima al principio y fin del mismo. El estudio fue realizado en el lado parético, el contralateral y en un grupo control de voluntarios sanos, comparándose los resultados de los 3 grupos. Las curvas de frecuencia y duración del lado parético, el sano y el control mantuvieron las mismas tendencias, así como fue homologable el comportamiento de la onda $M$ máxima en los 3 casos, lo cual indica que en la espasticidad, debido a daño de la via piramidal, al igual que en la población normal, bajo las presentes condiciones de estudio, es el factor central el condicionante principal en el desarrollo de fatiga muscular. 


\section{SUMMARY}

\section{Muscular fatigue mechanisms in pyramidal lesions.}

Muscular fatigue mechanisms were partially analyzed in patients with pyramidal lesions by using a computer device on line with a conventional electromyograph. Both the normal and the affected side were compared with control groups. The recordings were done throughout a ten minutes period and the maximal $M$ wave was also measured at the starting and at the end of the procedure. The findings suggested that central events play a major role in the development of muscular fatigue in spastic muscles due to pyramidal damage.

\section{REFERENCIAS}

1. CAMPbell, M. J.; MC COMAs, A. J. \& PETito, F. - Physiological changes in a eing muscles. J. Neurol. Neurosurg. Pshychiat. 36:174, 1973.

2. MC COMAS, A. J.; SICA, R. E. P. \& UPTON, A. R. M. - Functional compensation in partially denervated muscles. J. Neurol. Neurosurg. Psychiat. 34:453, 1971.

3. MC COMAS, A. J.: SICA, R. E. P.; UPTON, A. R. M. \& AGUILERA, N. Functional changes in motoneurones of hemiparetics patients. J. Neurol. Neurosurg. Psychiat 36:183, 1973.

4. PANIZZA, Marcela E.; COHEN, L. G.; SANZ, Olga; REY, R.; SCHUTZ, C. Evaluación del factor central y periférico en fatiga muscular. Arq. Neuro-Psiquiat., (São Paulo) 41:241, 1983.

5. ROSENFALCH, A. \& ANDREASSENN, S. - Impaired regulation of force and ing pattern of single motor units in patients with spasticity. J. Neurol. Neurosurg Psychiat 43:907, 1980.

6. SICA, R. E. P. \& SANZ, O. P. - An electrophysiological study of the functional changes in the spinal motoneurones of hemiparetics patients. Electromyograph clin. Neurophysiol. 16:419, 1976.

7. TANG, A. \& RYMER, Z. - Abnomal force-EMG relations in paretic limos of hemiparetic human sujets. J. Neurol. Neurosurg. Psychiat. 44:690, 1981.

Sacción de Electroneurofisiología Clinica. Departamento de Neurologí. Hospital Ramos Mejia. Buenos Aires, Argentina. 